\title{
Monodromy of the LiNC/NCLi molecule
}

\author{
M. Joyeux ${ }^{2}$ \\ Laboratoire de Spectrométrie Physique, Université Joseph Fourier - Grenoble I, \\ UMR 5588 du CNRS, Bô̂te Postale 87, 38 402 St. Martin d'Hères, France \\ D. A. Sadovskií ${ }^{1}$ \\ Université du Littoral, UMR 8101 du CNRS, 59140 Dunkerque, France \\ J. Tennyson ${ }^{3}$ \\ Department of Physics and Astronomy, University College London, \\ Gower Street, London, WC1E 6BT, United Kingdom
}

\begin{abstract}
Using the potential surface of Essers, Tennyson, and Wormer in [Chem. Phys. Lett. 89 (1982) 223] we show that the system of bending vibrational states of the isomerizing molecule LiNC/NCLi has monodromy. On the basis of a deformed spherical pendulum model we explain dynamical and geometric reasons of this phenomenon and of its absence in the similar system $\mathrm{HCN} / \mathrm{CNH}$.
\end{abstract}

Monodromy is an interesting property of certain quantum systems whose levels cannot be labelled globally by one set of quantum numbers. More precisely, even though such systems have no obvious subsystems, each with its own set of quantum numbers, quantized energy cannot be expressed as one smooth function of any quantum numbers. Dynamical meaning of this phenomenon can be uncovered $[1,2]$ by analyzing monodromy of the corresponding classical analogue. For the details of the classical theory see [3] and references therein.

Monodromy is not uncommon. It has been found in several fundamental atomic and molecular systems, notably the perturbed hydrogen atom [4], Stark effect in rotating dipolar molecules [5], rotating quasi-linear triatomic molecules [6], systems with coupled angular momenta [7], $\mathrm{H}_{2}^{+}$[8]. Floppy triatomic molecules with linear equilibrium configuration(s) is another candidate

1 sadovski@univ-littoral.fr, corresponding author

2 marc.joyeux@ujf-grenoble.fr

3 j.tennyson@ucl.ac.uk

Preprint submitted to Chem. Phys. Lett. $\quad$ DS 9/03, printed: 21 September 2003 
for a system with monodromy [9]. In this letter we describe monodromy of $\mathrm{LiNC} / \mathrm{NCLi}$. We compare this system to $\mathrm{HCN} / \mathrm{CNH}$, which we have analyzed recently in [10].

Our study of LiNC/NCLi uses the two-dimensional potential [14] which gives the energy of the LiNC configurations as function of the bending angle $\gamma$ and Li-to-NC stretch distance $R$ for the length $r$ of the $\mathrm{N}-\mathrm{C}$ bond fixed at 2.186 bohr. We assume approximate separability of the two degrees of freedom corresponding to overall rotations of the molecule about the two axes which are roughly orthogonal to the $\mathrm{N}-\mathrm{C}$ axis and we only consider states without such rotations. For these states the total angular momentum $J$ equals the angular momentum $\ell$ induced by the rotations of $\mathrm{Li}$ about axis $\mathrm{N}-\mathrm{C}$. The other bending degree of freedom is described by $\gamma$, and the remaining third degree is stretching vibrations of $R$. We find the minimum energy path $R(\gamma)$ and consider small stretching oscillations about this path. The frequency of these oscillations and the bending mode frequencies are incommensurate. This allows us to average the stretching mode out using the quantum analogue [13] of the canonical perturbation theory $[11,12]$ and introduce stretching quantum number $n_{R}$. We obtain the reduced effective bending mode Hamiltonian $H_{\ell, n_{R}}\left(p_{\gamma}, \gamma\right)$ and use it for the ground stretching state with $n_{R}=0$. We study all $\ell$-states and the advantage of our approach is that the value of quantum number $\ell$ is predefined.

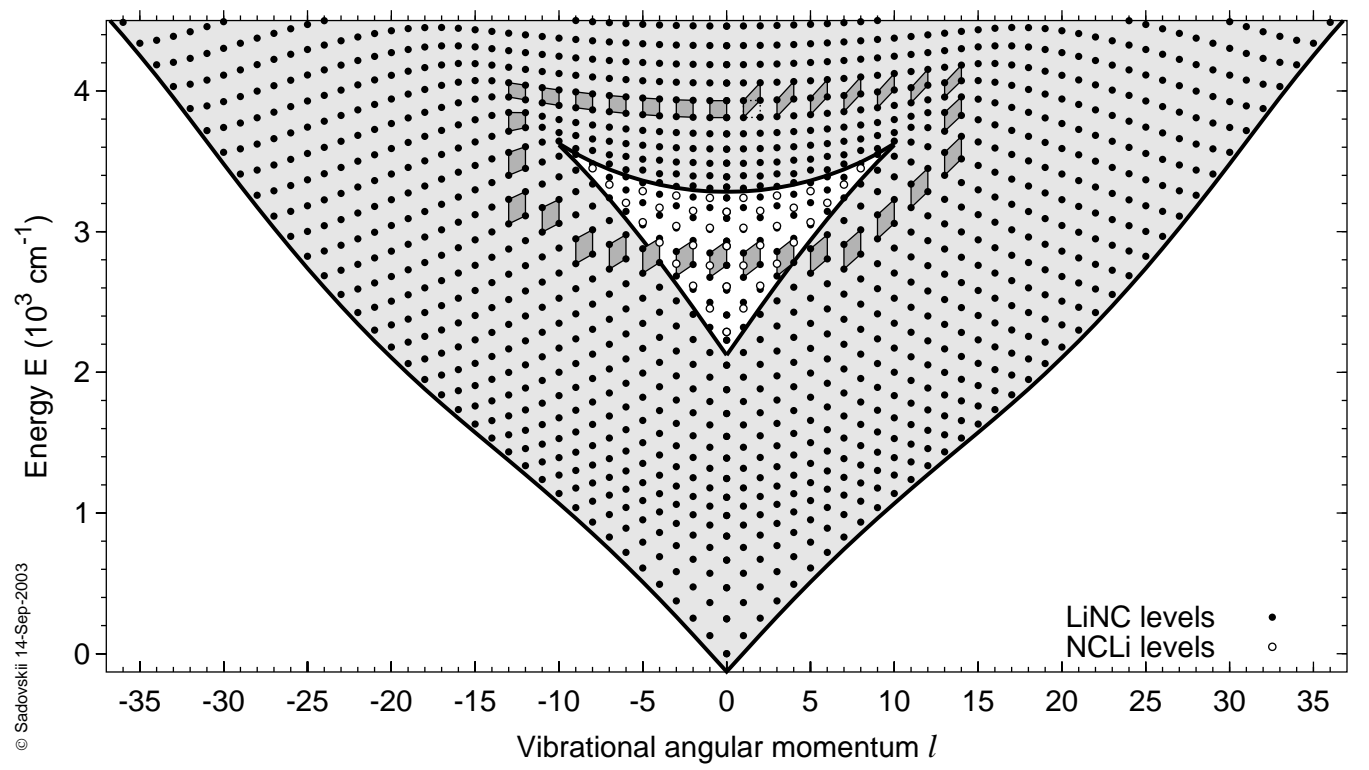

Fig. 1. Energy-momentum diagram of the LiNC/NCLi system computed for the potential in [14]. Filled and hollow circles show levels attributed to the LiNC and NCLi minimums; bold solid lines show energies of classical relative equilibria.

Using $H_{\ell}\left(p_{\gamma}, \gamma\right)$ we computed quantum energies for each $\ell$. The energies (of the $\ell=0$ band origins?) are in good agreement with full quantum calculations in [15] and this justifies our approach (at least for small $\ell$ ?). We also found 
energies of classical relative equilibria which correspond to Li rotating about the axis of $\mathrm{N}-\mathrm{C}$ at constant $\gamma$ and can be found as equilibria of the classical analogue system with Hamiltonian $H_{\ell}\left(p_{\gamma}, \gamma\right)$ for $\ell \neq 0$. For $\ell=0$ we have two stable linear equilibria of which LiNC is the lowest in energy and a barrier separating the two wells.

Results of our computation are presented in the form of the energy-momentum diagram shown in fig. 1. This diagram should be considered as a two-leaf surface. The leafs are bounded by the energies of relative equilibria. In fig. 1, the smaller NCLi leaf and the larger LiNC leaf are shaded white and gray respectively. The NCLi leaf is closed, while the LiNC leaf is unbounded from above. The NCLi leaf is glued along its upper boundary to the LiNC leaf. This latter has therefore a critical "cut" surrounded by regular values of the energy-momentum map.

The energies of quantum states form a lattice in each of the leaves. As the labels imply, the two leaves represent states localized near the LiNC and NCLi equilibria, but the LiNC leaf also includes delocalized states which correspond to Li making complete tours in $\gamma$ about NC. While NCLi states are all "locked" in their leaf, there is nothing to separate localized LiNC states from delocalized states at sufficiently high $\ell$. Of course, the energy-momentum diagram has only two dimensions and the two leaves and their lattices are shown overlapped in fig. 1.

We determine the monodromy of LiNC/CNLi using the elementary cell method of Zhilinskií [7] which blends the initial approach in [1,2] and the methods used to describe the defects of crystal lattices. We begin by choosing a nearly square elementary cell of the LiNC lattice at $\ell=0$ and energy $E \approx 4000 \mathrm{~cm}^{-1}$, i.e., above the singular cut, see fig. 1 . This cell reflects our choice of local quantum numbers, it defines neighbour states whose one or both quantum numbers differ from those of the given state by 1 . We continue our elementary cell along the path which goes counterclockwise around the cut in small steps. Note that in the overlap area of the LiNC and NCLi leaves, we can only use LiNC states (black dots in fig. 1). At each step the next cell is defined unambiguously by the current cell, i.e., by the current choice of local quantum numbers. However, when we come back to $\ell=0$ and $E \approx 4000 \mathrm{~cm}^{-1}$ after one tour, the final cell 1 does not correspond to the original cell $\square$. The transformation is given by the monodromy matrix $M=\left(\begin{array}{ll}1 & 1 \\ 1 & 0\end{array}\right)$, the monodromy is 1 (the off-diagonal element of $M$ ). Note that a very similar phenomenon has been recently studied in [8], where it was somewhat misleadingly called "island monodromy".

The origins of monodromy of LiNC/NCLi can be best understood after comparing the bending motion of LiNC/NCLi to that of the spherical pendulum 
in [3, Chap. IV.3]. This latter can be represented as a motion of a particle placed in a linear potential $V(z)=z$, e.g., in the field of gravity, and constrained to a sphere. It has one stable equilibrium at the bottom of the sphere that corresponds to the minimum energy and one unstable equilibrium; both equilibria are at $\ell=0$. In the image of the energy-momentum map, the unstable equilibrium together with all its homoclinic orbits is represented by an isolated critical point, shown by a black circle dot in fig. 2, left.
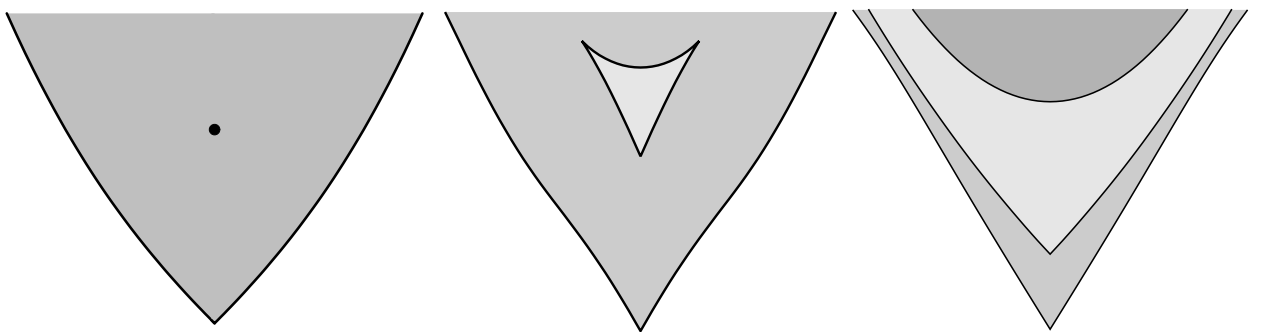

Fig. 2. Energy-momentum values (left to right) of the spherical pendulum [3, Chap. IV.3], LiNC/NCLi $\left(\epsilon<\frac{1}{3}\right)$, this work, and HCN/CNH $\left(\epsilon>\frac{1}{3}\right)$ [10].

Comparing the above to the molecular sytems LiNC/NCLi or $\mathrm{HCN} / \mathrm{CNH}$, we notice two principal differences: the potential and the "shape". Both linear equilibria of these molecules are stable. This can be described qualitatively if the linear potential of the pendulum is replaced by a quadratic potential $V(z)=z+c z^{2}$. The shape is given by the distance $R$ between $\mathrm{Li}($ or $\mathrm{H}$ ) and the $\mathrm{CN}$ diatom at different values of the bending angle $\gamma$ along the minimum energy path of the potential surface. In the spherical pendulum $R$ is fixed. As shown in fig. 3, the molecules are shaped more like a peanut because $\mathrm{Li}$ (or $\mathrm{H}$ ) can get closer to the diatom in the T-configuration when $\gamma \approx \frac{1}{2} \pi$. We can model such shape using $R(\gamma)=R_{\max }\left(1-\epsilon \sin ^{2} \gamma\right)$ where $\epsilon \geq 0$ is the asphericity parameter.
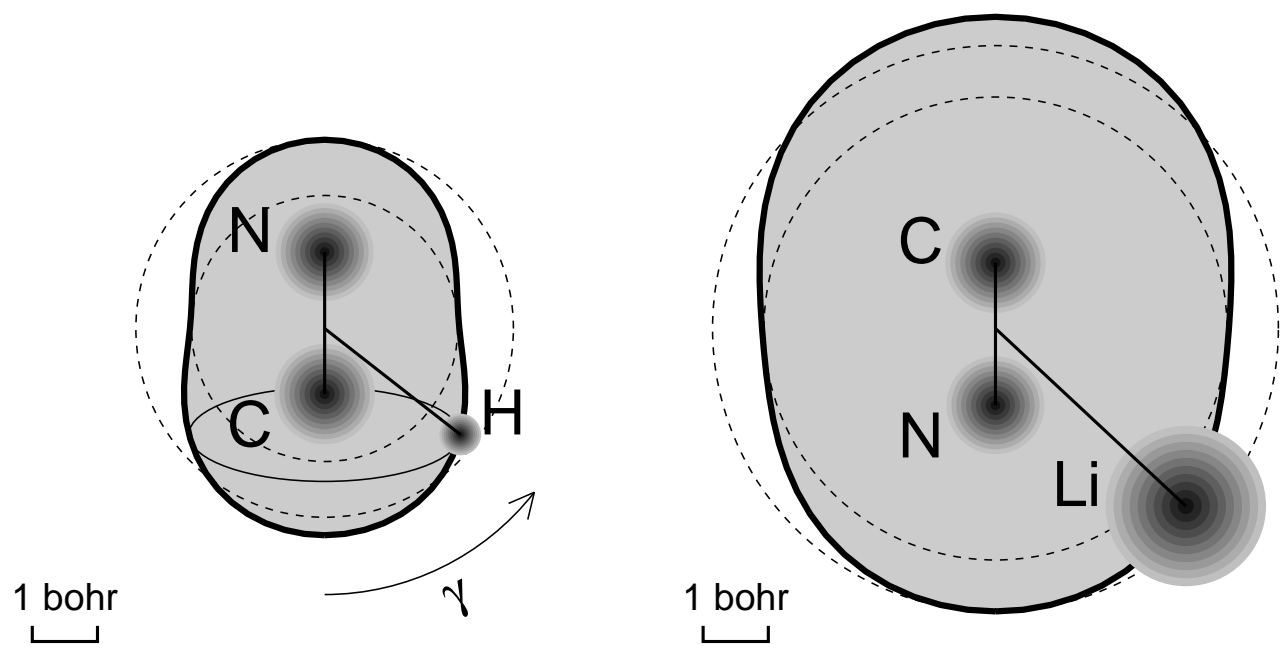

Fig. 3. Geometry of the HCN/CNH and LiNC/NCLi systems computed using the potentials in [16] and [14] respectively. 
When the deformation of the original spherical pendulum system is small, i.e., for $c$ and $\epsilon$ small compared to 1, the energy-momentum map should not change a lot. Concrete computations [10] show that the isolated singular point (fig. 2, left) becomes a small triangular "island" leaf (fig. 2, center) glued to the main leaf along its upper boundary as we already explained before. However, when $\epsilon>\frac{1}{3}$ this new leaf, which represents the motion localized near the second stable linear equilibrium, becomes unbound from above at sufficiently high $\ell$ (fig. 2, right). At such large $\epsilon$ the singular cut, along which the two leafs are glued together, is no longer a finite segment. This cut now separates the energy-momentum values of the motion localized near the equilibria from those of the delocalized motion ("rotation" about the diatom). As a result, we can no longer loop around the cut in the domain of the regular energymomentum values, as we did for LiNC/NCLi (fig. 1). Consequently, systems with $\epsilon>\frac{1}{3}$ do not have monodromy.

The value of $\epsilon=\frac{1}{3}$ has a remarkably simple geometric meaning. At this value, the shape of the system bifurcates so that for $\epsilon>\frac{1}{3}$ the molecule develops a "waste". A simple rough estimate gives $\epsilon=0.26$ for $\mathrm{LiCN}$ and $\epsilon=0.36$ for $\mathrm{HCN}$. Indeed, the $\mathrm{HCN} / \mathrm{CNH}$ system (fig. 3, left) has a small waste, while $\mathrm{LiNC} / \mathrm{CNLi}$ is convex. So the conclusion in [10] that the $\mathrm{HCN} / \mathrm{CNH}$ molecule has no monodromy (fig. 2, right) comes as no surprise.

In the present letter, we have considered the vibrational subsystem of LiNC that includes two stretching and two bending degrees of freedom and have shown that this subsystem has monodromy. Before extending this statement to the complete system, we should investigate the interaction between these degrees of freedom and the two remaining degrees related to overall rotations of the molecule. In our treatment based on [11-13] we neglected these rotations by excluding the respective part of the kinetic energy. While this might be an appropriate approximation in $\mathrm{HCN} / \mathrm{CNH}$ where $\mathrm{H}$ does not influence significantly the inertia tensor, it becomes more problematic in LiNC/NCLi because $\mathrm{Li}$ is heavier and moves at larger distances. The complete rotation-vibration system requires, therefore, further study.

As the number of known fundamental quantum systems with monodromy grows, it becomes important to understand how this phenomenon can be manifested and exploited experimentally. Such study involves additional theoretical expertise in semiclassical theory, wavepacket techniques, and geometric phase, as well as knowledge of modern experimental methods in the time domain. 


\section{Acknowledgement}

This work was made possible by the CNRS research position granted to D.S. for the 6 month in 2003. D.S. and J.T. acknowledge support by the EU network project Mechanics and Symmetry in Europe (MASIE), contract HPRN-CT2000-00113.

\section{References}

[1] R. H. Cushman, J. J. Duistermaat, Bull. Am. Math. Soc. 19 (1988) 475.

[2] S. Vũ Ngọc, Commun. Math. Phys. 203 (1999) 465.

[3] R. H. Cushman, L. Bates, Global aspects of classical integrable systems, Birkhauser, Basel, 1997.

[4] D. A. Sadovskií, R. H. Cushman, Physica D 142 (2000) 166.

[5] I. N. Kozin, R. M. Roberts, J. Chem. Phys. 118 (2003) 10523.

[6] M. S. Child, T. Weston, J. Tennyson, Mol. Phys. 96 (1999) 371.

[7] D. A. Sadovskií, B. I. Zhilinskií, Phys. Lett. A 256 (1999) 235.

[8] H. Waalkens, A. Junge, H. R. Dullin, J. Phys. A. Math. Gen. 36 (2003) L307.

[9] M. P. Jacobson, M. S. Child, J. Chem. Phys. 114 (2001) 262.

[10] K. Efstathiou, M. Joyeux, D. A. Sadovskií, Phys. Rev. A (2003) in press.

[11] D. Sugny, M. Joyeux, J. Chem. Phys. 112 (2000) 31.

[12] D. Sugny, M. Joyeux, E. L. Sibert III, J. Chem. Phys. 113 (2000) 7165.

[13] M. Joyeux, D. Sugny, Can. J. Phys. 80 (2002) 1459.

[14] R. Essers, J. Tennyson, P. E. S. Wormer, Chem. Phys. Lett. 89, 223 (1982).

[15] J. R. Henderson, J. Tennyson, Mol. Phys, 69 (1990) 639.

[16] T. van Mourik, G. J. Harris, O. L. Polyansky, J. Tennyson, A. G. Császár, P. J. Knowles, J. Chem. Phys. 115 (2001) 3706. 